\title{
Gender-specific hypertension in mice deficient in the alphal subunit of soluble guanylate cyclase
}

\author{
Patrick Sips*1, Emmanuel Buys ${ }^{1}$, Robrecht Thoonen ${ }^{1}$, Anje Cauwels ${ }^{1}$, \\ Elke Rogge ${ }^{1}$, Maureen Van Den Hemel ${ }^{1}$, Mieke Dewerchin ${ }^{2}$ and \\ Peter Brouckaert ${ }^{1}$
}

\begin{abstract}
Address: ${ }^{1}$ Department of Molecular Biomedical Research, Molecular Pathology and Experimental Therapy unit, Flanders Interuniversitary Institute for Biotechnology (VIB-1), Ghent University, Zwijnaarde, Belgium and 2Department of Transgene Technology and Gene Therapy (VIB-3), Catholic University of Leuven, Belgium
\end{abstract}

Email: Patrick Sips* - Patrick.Sips@dmbr.ugent.be

* Corresponding author

from 2nd International Conference of cGMP Generators, Effectors and Therapeutic Implications Potsdam, Germany, 10-12 June, 2005

Published: 16 June 2005

BMC Pharmacology 2005, 5(Suppl I):P52 doi:10.1186/I47I-22I0-5-SI-P52

Soluble Guanylate Cyclase (sGC) is a heterodimeric heme containing enzyme of which two active isoforms have been identified: alpha1beta1 and alpha2beta1. It catalyzes the formation of the intracellular messenger molecule guanosine 3'-5'-cyclic monophosphate (cGMP) from guanosine $5^{\prime}$-triphosphate (GTP), and is a major effector protein of the ubiquitous signaling molecule Nitric Oxide (NO). As such it is an important central mediator in the $\mathrm{NO} / \mathrm{cGMP}$ pathway, and is involved in the regulation of a number of physiological processes such as neurotransmission, platelet reactivity and particularly smooth muscle relaxation; thereby regulating blood pressure, gastrointestinal motility and genital erection. Dysfunction of this pathway is involved in the pathogenesis of several diseases.

We chose to use transgenic technology to study the potential value of the sGC isoforms as drug targets. Besides obtaining homologous recombinant ES-cells for both a (conditional) sGCalpha2 knockout and a sGCbeta1H105P knockin, which can (presumably) no longer be activated by NO, we were able to obtain sGCalpha1 full and conditional knockouts making use of the Cre/LoxP system. Cardiovascular phenotyping of these mice showed a genderspecific hypertension in the male knockouts (see Figure 1 ), which only appears after 12 weeks of age. This hypertensive phenotype seems very interesting for cardiovascular medicine, seeing as it reflects the situation in humans where males are more prone to hypertension and ensuing cardiovascular accidents as compared to premenopausal women.

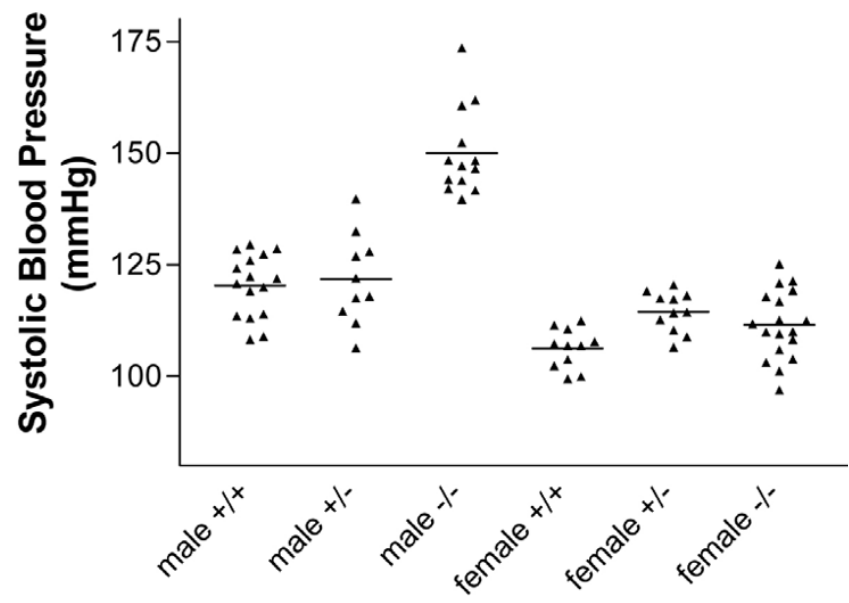

Figure I

Gender-specific hypertension in sGCalphal knockout mice. Blood pressure measurements on male and female wildtype $(+/+)$, heterozygous (+/-) and sGCalphal knockout (-/-) mice of at least 16 weeks old. Male knockout mice are hypertensive (ANOVA: $p<0,000 I$ ). 